\section{Digital in-line holography}

\author{
H.J. Kreuzer" and R.A. Pawlitzek" \\ - Department of Physics, Dalhousie University, Halifax, Canada \\ "IBM Zurich Research Laboratory, Switzerland
}

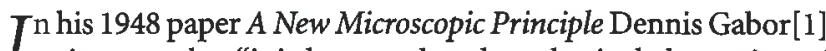
$I$ points out that "it is known that the spherical aberration of electron lenses sets a limit to the resolving power of electron microscopes at about $5 \AA$.... Prospects of improvement are... aggravated by the fact that the resolution limit is proportional to the fourth root of the spherical aberration." He continues "The new microscopic principle described below offers a way around this difficulty, as it allows one to dispense altogether with electron objectives." He called it "electron interference microscopy" what later became holography.

The principle of holography is most easily explained with Gabor's original implementation as illustrated in Figure 1. A spherical wave of wavelength $\lambda$, emanating from a "point" source of linear dimensions of the order of the wavelength, illuminates an object, typically a distance of a few thousand wavelengths from the source, and forms a highly magnified diffraction pattern on a screen much further away, see Fig.1. We denote by $A(r, t)$ the wave amplitude (the wave function for electrons or the electric field vector for photons) and split it into the unscattered reference wave, $A_{\text {ref }}(\mathbf{r}, \mathrm{t})$, and the wave, $A_{\text {scat }}(r, \mathrm{t})$, scattered by the object. Ideally the reference wave emanating from the source is a spherical wave, $A_{\text {ref }}(\mathbf{r}, \mathrm{t})=\exp (i k r) / r$ where $k=2 \pi / \lambda$ is the wave number. In an experiment this spherical wave is modified by the emission cone of the source. However, this can be accounted for by constructing, from the intensity, $|A(\mathbf{r}, \mathrm{t})|^{2}$, the contrast image

$$
\begin{aligned}
\tilde{I}(\mathbf{r}) & =\left|A_{r e f}(\mathbf{r}, t)+A_{s c a t}(\mathbf{r}, t)\right|^{2}-\left|A_{r e f}(\mathbf{r}, t)\right|^{2} \\
& =\left[A_{\text {ref }}^{*}(\mathbf{r}, t) A_{s c a t}(\mathbf{r}, t)+A_{r e f}(\mathbf{r}, t) A_{s c a t}^{*}(\mathbf{r}, t)\right]+\left|A_{s c a t}(\mathbf{r}, t)\right|^{2}
\end{aligned}
$$

We will refer to the first term (in brackets) in (1), linear in the scattered wave, as the "holographic diffraction pattern" because it arises from the superposition of the interference terms between the unscattered reference wave from the source and the scattered wave from the object. The second term in (1) contains the interference between the scattered waves. We will refer to it as the "classical diffraction pattern". Holographic diffraction dominates the images for small objects; loosely speaking "small" means that the object should only block a fraction of the cone of radiation recorded on the screen. Even if these objects are opaque, we are still in the regime of holography, and will be able to recover the outline or shape of the object. As the scattered wave amplitude grows because more of the incoming reference wave is blocked

$\begin{array}{|ll|}\text { Screen } & \text { Fig. 1: Schematic } \\ \text { Point source } & \text { of in-line holography: } \\ \text { A coherent wave of } \\ \text { radiation emanating } \\ \text { from the point source } \\ \text { partially scatters off } \\ \text { an object creating a } \\ \text { highly magnified } \\ \text { hologram on a screen. }\end{array}$

out by larger objects, classical diffraction becomes more important. When it eventually dominates the image, we are in the regime of classical wave optics.

A hologram is just a complicated interference pattern from which little information about the shape and structure of the object can be obtained by simple inspection. What object, as an example, produced the hologram in panel (a) of Fig.2? Holography was therefore conceived by Gabor as a two-step process: first, a hologram must be recorded, and second, reconstruction must yield an "image" of the object, i.e. the intensity of the scattered wavefront at the object. The reason why the second step is possible is the fact that the holographic information is linear in the scattered wave so that an inversion of the scattering process is feasible. This, Gabor envisaged, could be achieved by recording the hologram photographically on a transparent plate and then "looking" through the hologram from the back. As Gabor put it: "One must expect that looking through such a properly processed diagram one will see behind it the original object, as if it were in place." Gabor tested this idea with visible light but the quality of the reconstruction was not overwhelming. More importantly, the application in electron microscopy needed modification for two reasons: (1) A photographic plate is not transparent to electrons, and (2) using the same wavelength for the reconstruction would not yield any magnification. Therefore Gabor suggested to use visible light for the reconstruction after scaling up all dimensions in the ratio of light waves to electron waves, that is, by about a factor 100,000 .

Although attempts at electron holography were made early on, their success was very limited, essentially because the condenser lenses for electrons at the time were so poor that no focus of the size of the electron wavelength of much less than angstroms could be achieved. A breakthrough came in the late 1980's when Fink and collaborators showed that an ultrathin metal tip with one or a few atoms at its apex serves as a "point" source with a virtual source size of atomic dimensions for a coherent beam of electrons with energies in the $10-200 \mathrm{eV}$ range. A new kind of microscopy with point sources, now called Low Energy Electron Point Source (LEEPS) microscopy, evolved from combining the technological tools of scanning probe microscopy with new ideas in projection microscopy. We refer the reader to a recent review article with further details and references.[]

In the optical realm holography took off in the early 1970's after the availability of lasers. Although holography with spherical waves, as originally proposed by Gabor and now called in-line holography, is the simplest realization of the holographic method, because it works without lenses, its applications had been limited until recently due to the fact that reconstruction of the object image with another wave is not practical. To avoid this problem various schemes of off-line holography have been developed in which a laser beam is split to provide an undisturbed reference wave while the other beam is focussed onto the object. There are too many variations on this scheme to allow an adequate overview here and the reader is referred to two books $[4,5]$ in which the principles and the practical implementations are described in detail. The remainder of this article will deal exclusively with our work on digital in-line holography with numerical reconstruction. ${ }^{1}$

In digital in-line holography-DIH-the hologram is recorded by a detector array, such as a CCD camera for photons, and transferred to a computer in which the reconstruction is done numerically. The role of reconstruction is to obtain the threedimensional structure of the object from the two-dimensional hologram on the screen, or, in physical terms, to reconstruct the 
wave front at the object. This can be achieved via a KirchhoffHelmholtz transform $[2,6]$

$$
K(\mathbf{r})=\int_{S} d^{3} \xi \widetilde{I}(\boldsymbol{\xi}) \exp [2 \pi i \boldsymbol{\xi} \cdot \mathbf{r} /(\lambda \xi)]
$$

in which the integration extends over the two-dimensional surface of the screen with coordinates $\xi=(X, Y, L)$ where $L$ is the distance from the source (pinhole) to the center of the screen (CCD chip); and $\tilde{I}(\xi)$ is the contrast image (hologram) on the screen obtained by subtracting the images with and without the object present. The function $K(\mathrm{r})$ is significantly structured and different from zero only in the space region occupied by the object. By reconstructing the wave front $K(\mathbf{r})$ on a number of planes at various distances from the source in the vicinity of the object, a three-dimensional image can be built up from a single two-dimensional hologram. $K(\mathbf{r})$ is a complex function and one usually plots its magnitude to represent the object, although phase images are also available. For the numerical implementation of the transform we have developed a fast algorithm that evaluates $K(\mathbf{r})$ without any approximations. It is incorporated in a selfcontained program package, originally developed for electron holography, called LEEPS, that not only performs the numerical reconstruction but also all other procedures connected with data management and visualization [7].

In holography, the term 'reconstruction' is used to obtain the function $K(\mathbf{r})$ from the hologram. The plot of $|K(\mathbf{r})|$ on a twodimensional plane, which we will call a 2-D holographic reconstruction, perpendicular to the optical axis is equivalent to one in-focus image taken in a conventional compound microscope. In DIH one can generate a stack of 2-D holographic reconstructions from a single hologram. Combining such a stack will result in a three-dimensional image of the object; this latter step is usually referred to as 3-D reconstruction.

The experimental setup for optical DIH, following the schematic of Fig.1, is very simple: a laser is focussed onto a pinhole, of a diameter of the order of the wavelength, which acts as the "point source" from which a spherical wave of wavelength $\lambda$, emanates. The wave illuminates an object, in our setup a few millimeters from the pinhole, and forms a geometrically magnified diffraction pattern on a screen, in our case a CCD chip, a few centimeters away. If the scattered wave, shown by dotted line in Fig.1, from the object is small compared with the unscattered reference wave, the interference pattern on the screen constitutes a hologram, linear in the scattered wave. The hologram is stored as a digital image in a computer for reconstruction.

To establish DIH as a new microscopy it is important to make detailed comparisons between images from conventional compound light microscopy and those obtained with DIH. For this purpose we used a the setup in the lower panel of Fig. 1 in which a standard inverted compound microscope is used to obtain a digital record of the bright-field image seen through the ocular in the normal way. To record a hologram of the same sample area, a pinhole (typical diameter 1-5 $\mu \mathrm{m}$ ) was placed between the objective lens and the sample such that the spherical light waves from the pinhole passed through the selected sample area. The laser light that illuminated the pinhole was introduced through a side port in the microscope and was directed towards the objective lens and pinhole via a movable mirror. The hologram was recorded with a CCD camera that was supported on the microscope work plate and centered over the pinhole and the selected area of the sample. The distance of the CCD camera from the pinhole, typically a few centimeters, was adjusted to capture all interfer-

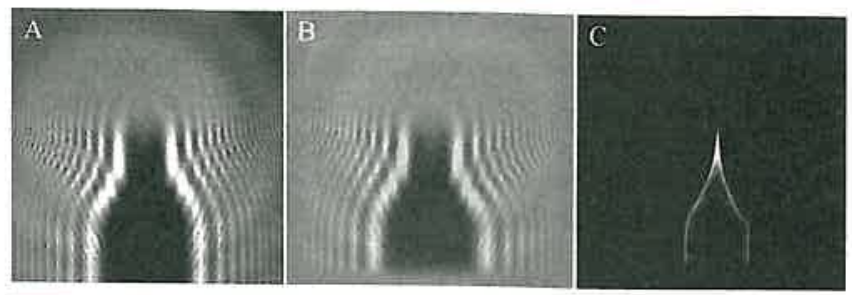

Fig. 2: A: Hologram of an Scanning Tunneling Microscope tip, $B$ : the contrast image and $C:$ its reconstruction.

ence fringes of the hologram that could be resolved with enough pixels. The pinhole was mounted on an X-Y micrometer stage and could be moved out of the way to image the sample in bright field. As we mentioned above the input to the reconstruction formula (2) is the contrast image for a perfectly spherical incoming wave. It transpires that perfecting this image is the hardest part in the practical implementation of DIH. Guided by the experience in photoelectron and LEED holography we have implemented the following procedure:

(i) Record digitally the hologram of the object, giving a matrix $I_{n m}$ of the intensity recorded on the CCD chip, where $n$ and $m$ enumerate the pixels in the $\mathrm{x}$ - and $\mathrm{y}$-directions.

(ii) Remove the object and record digitally the intensity matrix $I_{n m}^{(0)}$ of the illuminating laser.

(iii)Construct numerically the contrast image, corrected for the intensity variations in the primary laser beam, $\tilde{I}_{n m}=\left(I_{n m}-I_{n m}^{(0)}\right) / \sqrt{ } I_{n m}^{(0)}$. By this procedure almost all imperfections in the laser source are eliminated. Indeed, this step minimizes the quality requirements on the laser itself, as long as the laser is sufficiently stable to capture identically both images. We have checked this by comparing the contrast images obtained with (i) a high quality laser and (ii) a cheap laser pointer. The results in reconstruction are of comparable quality.

The contrast image, an example is given in panel (b) of Fig.2, is next used in the reconstruction algorithm, based on the transform (2), to produce a series of 2-D holographic reconstructions at different distances from the source, i.e. the pinhole. Because the source-object distance can be measured directly with an accuracy of only a millimeter or so, it is necessary to zoom in with a series of reconstructions at different distances, displayed in the LEEPS program package as a film, to achieve accuracy at the submicron level.

As a first application we show, in panel (c) of Fig.2, the reconstruction of the hologram in panel (b): it is an the tip of a Scanning Tunneling Microscope; the end of the tip has a diameter of less than $1 \mu \mathrm{m}$.

To demonstrate the state of the art in optical DIH we show in Fig. 3 holograms and images of latex microspheres having a mean diameter of $1 \mu \mathrm{m}$. The spheres (refractive index 1.59 at $589 \mathrm{~nm}$ ) were mounted in a thin layer of gelatin between a microscope slide and a glass coverslip. A hologram, i.e. the intensity matrix $I_{n m}$ taken with a blue laser, is shown in panel A. To obtain the intensity matrix of the light source, $I_{n n}^{(0)}$, the slide was moved sideways to an area that contained only gelatin but no spheres. This reference hologram was subtracted from the hologram in panel A to obtain the contrast hologram, panel B. In panel C we show a 2-D holographic reconstruction from the original, uncorrected hologram of panel A, taken through the equatorial plane of the spheres. The image clearly resolved all spheres but also shows a 
substantial noise level. Starting however, from the contrast hologram, panel B, gives a perfect reconstruction, panel D. Indeed, this image is better than a bright field image taken with the compound microscope for which diffraction rings already show up.

The power of DIH is that image information is not limited to a plane but is truly three-dimensional. 2-D holographic reconstructions can thus be performed from a single hologram at a series of selected object depths and the $3-\mathrm{D}$ reconstruction of the object can be generated from such a stack of 2-D reconstructions. We have done this for the latex spheres. Panel E shows the spheres in a 3-D reconstruction, viewed as if along the optical axis, that was obtained from a stack of 15 consecutive 2-D hologram reconstructions, $1 \mu \mathrm{m}$ apart. In panel $\mathrm{F}$, the same stack of 15 reconstructed sections was viewed as if along one axis of the section plane, to provide a side view of the spheres. Viewed from this direction, all spheres lie nearly in the same plane (as the sample geometry requires) and the spheres have again circular profiles. The two spheres that were in contact appear to occupy slightly different positions perpendicular to the sample plane, information that could not be obtained from conventional compound light microscopy alone. A detailed account of these studies is given elsewhere.[9]

DIH has by now been developed into a new microscopy in biology [8]. As an example, we show reconstructed images of a unicellular marine plant cell, the diatom Ditylum brightwellii, mounted unstained in gelatin (Fig. 4). Images obtained using DIH (Figs. 4E, F) are compared with a bright-field transmitted-light image (Fig. 4A, a differential interference (DIC) microscopy image (Fig. 4B) using a conventional compound light microscope, and two views of a stack of images obtained by laser scanning confocal microscopy (LSCM) and reconstructed in 3-D using software (Figs. 4C, D). Note that all images are shown at the same magnification, using the same objective. Figs. $4 \mathrm{~A}$ and $4 \mathrm{~B}$ are sections, whereas Figs. $4 \mathrm{C}$ to $4 \mathrm{~F}$ are $3-\mathrm{D}$ reconstructions. It is clear that the fluorescence images of Figs. $4 \mathrm{C}$ and $4 \mathrm{D}$, originating mostly from the cell's chlorophyll-bearing plastids, contain only a

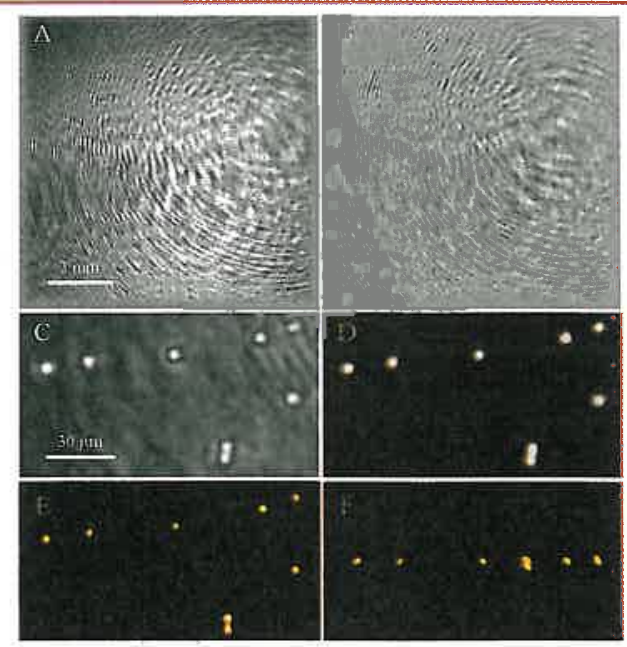

A Fig. 3: Latex microspheres ( $5 \mu$ m diameter) embedded in gelatin between glas plates. A: hologram (1024×1024 pixels), B: contrast hologram. $C$ and $D$ : reconstruction from $A$ and $B$, respectively, through the equatorial plane of the spheres. $(E)$ and (F) 3-D reconstruction from 15 2-D holographic reconstructions (of which $(D)$ is number 8); (E) viewed along the optical axis, and (F) viewed from the side. Pinhole - object distance $1.5 \mathrm{~mm}$, pinholeCCD chip distance $4.8 \mathrm{~cm}$, green laser $(\lambda=532 \mathrm{~nm})_{r} 2 \mu \mathrm{m}$ pinhole.
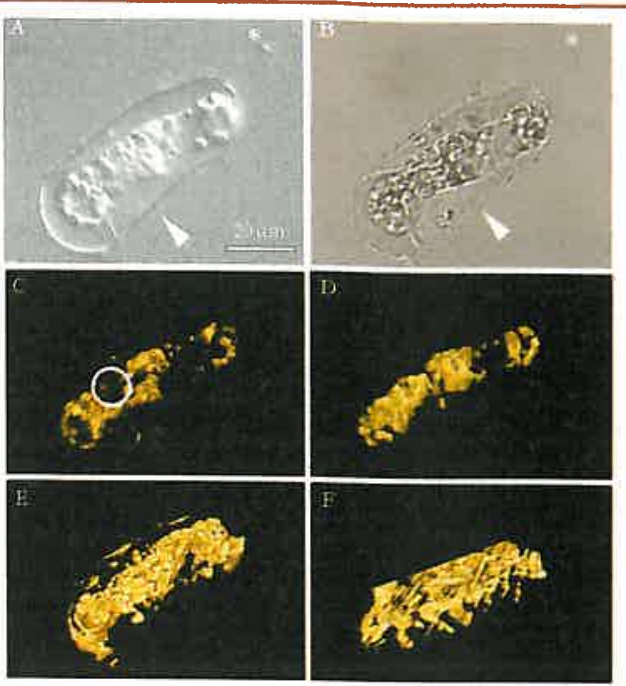

4. Fig. 4: Single cell of Ditylum brightwellii. (A) Differential interference microscopy; and (B) a bright-field image obtained in the transmission mode of a confocal microscope. (C, D) 3-D image stack of signal from specimen autofluorescence obtained by confocal microscopy and reconstructed from 23 slices at $1 \mu \mathrm{m}$ intervals, using the same specimen and objective as (B). Note contrast resolution of cell contents and absence of fluorescence in region enclosed by circle. (C) Viewed from same direction as in (A) and $(B)$; $(D)$ reconstruction rotated $90^{\circ}$ about the cell's long axis from the view in $(C)$. $(E, F)$ 3-D reconstruction of the corresponding DIH image stack. (E) Same view as (C), along the optical axis. (F) rotated view, perpendicular to (C), as in (D).

subset of the structures imaged by DIH in Figs. $4 \mathrm{E}$ and $4 \mathrm{~F}$. More complete evaluation of the $\mathrm{DIH}$ reconstructions can be made with reference to the original 2-D DIH images representing cuts through the cell. We refer the reader to an earlier paper where further details are given[8].

DIH can also be used successfully on macroscopic biological specimens, prepared by standard histological procedures, as for a histological section of the head of the fruit fly, Drosophila melanogaster. Such images reveal the structure of the pigmented compound eye, and different neuropile regions of the brain within the head cuticle including the optic neuropiles underlying the compound eye [8].

Because DIH offers a rapid and efficient approach to construct high-contrast 3-D images of a sample volume from a single hologram, a CCD camera having a fast capture rate allows us to record digitally from their successive positions the motion of particles in a sample volume, and subsequently to reconstruct images at selected depths so that the trajectory and speed of a collection of particles can be captured as 3-D data sets. To illustrate this feature of DIH, we recorded holographic images (Fig. 5A) of a live culture of the single-celled marine flagellate Tetraselmis (insert in Fig. $5 \mathrm{~A}$ ), contained in a $1 \mathrm{~mm}$ thick layer of salt water between a microscope slide and a coverslip. These algae are flattened and propel themselves by four anterior flagella. To obtain high-resolution DIH reconstruction images of the movement trajectories of the algae, we used the following procedure: i) A sequence of holograms $\left(h_{i}\right)$ was recorded at $0.06 \mathrm{sec}$ time intervals (the ratelimiting minimum interval for data transfer with our camera); ii) undesired background effects (such as the set of large concentric rings in Fig. 5A) were eliminated by subtracting consecutive hologram pairs, pixel by pixel, to generate new holograms, i.e. (h1-h2), 


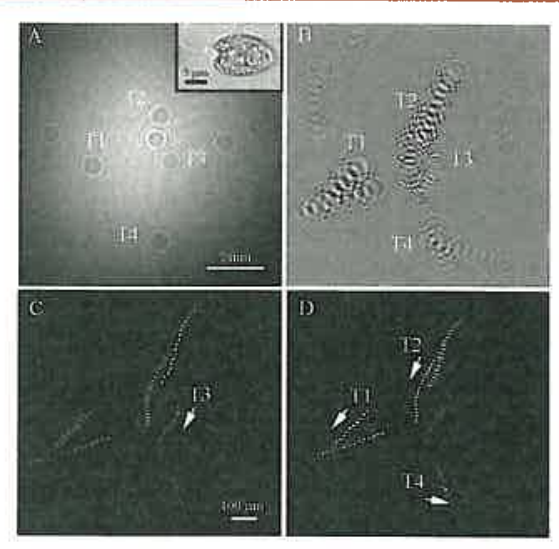

Aig. 5 : Holograms and reconstructed trajectories of the alga Tetraselmis in sea-water. A) Single hologram of many algae; inset: bright-field image of single alga, showing flagellae. B) Sum of 10 difference holograms (from a total of 20 ) of the trajectories of the algae in (A). (C-D) Reconstruction of the summed hologram (B) in two planes, $350 \mu \mathrm{m}$ apart. (Blue laser, $1 \mu \mathrm{m}$ pinhole, source to sample distance $2.5 \mathrm{~mm}$, source to CCD camera distance $20 \mathrm{~mm}$.)

(h3-h4), etc. (iii) The resultant difference pictures were summed (a few hundred holograms could be combined) into a single file, which now contained all holograms (albeit with alternate sign), yet had the same size as any single original hologram (Fig. 5B). Subtracting alternate holograms ensured that the dynamic range was not exceeded. The combined file was then reconstructed to obtain images at different depth planes in the water drop. Such images show the sequential positions at successive recording times of the algae contained in the sample volume. The trajectories of several algae in two such reconstructions are shown in two planes separated by $350 \mu \mathrm{m}$ (Figs. 5C and D). Twenty sequential positions clearly define the trajectories some of which are more or less confined to a plane but others dive in and out of the reconstruction plane shown, e.g. alga T3. Moreover some trajectories, sharply seen in one plane, are completely out of focus or even invisible in the other plane. Closer inspection also reveals that these algae sometimes rotate about their long axis when they change direction, the flattened shape alternately imaged edge on or en face. The access to 3-D information in holographic imaging is particularly well illustrated by the alga T3 (Fig. 5C). This alga comes to lie in the reconstruction plane but earlier images are progressively out of focus, indicating that the direction of motion had a large component perpendicular to the reconstruction plane. To obtain the complete trajectory, further reconstructions from the same hologram file would be made in as many planes as necessary without the need for further experiments. The complete stack can then be combined into a film of which the successive frames correspond to focussing at different depths as time goes on. Such a film can be viewed at http://www.physics.dal.ca/ -kreuzer. We emphasize the efficiency in data collection in our procedure for background removal and construction of summed holograms, which makes it possible to undertake high-resolution tracking of many particles in 4-D from just a single hologram data set. Since resolutions on the order of the wavelength of light have been achieved with DIH, tracking of organisms as small as bacteria should also be possible, as would the motion of plankton in water or microparticles in fluid flow or, at lower resolution, the aerial trajectories of flying insects.

To put the scope of 4-D tracking with DIH in perspective it is useful to think how this would be done in conventional microscopy: one would focus on a particular alga and, by rapid refocusing, would try to follow its motion recording images and focal positions. But this would only give information on the path of one alga. Contrast this with $\mathrm{DIH}$ where the motion of all algae in the sample volume can be reconstructed from a single file containing the time series of holograms without any need for further adjustments at all.

We conclude with a listing of the advantages of DIH:

1. Simplicity of the microscope: In-line holography is microscopy without objective lenses. The hardware required is a laser, a pinhole and a CCD camera.

2. Maximum information: A single hologram contains all the information about the three-dimensional structure of the object. The magnification is done in the computer.

3. Maximum resolution: Optimal resolution, of less than the wavelength of the laser, can be obtained easily.

4. Speed: The kinetics of the sample, such as the motion of many objects such as particulate matter in a fluid, living algae in water or organelle transport in a biological specimen, can be followed at the capture rate of the CCD chip.

5. Simplicity of sample preparation, particularly for biological samples where neither sectioning nor staining is required, means that living cells can be viewed.

Where do we go from here? We have hopefully made the point that DIH is now ready for routine applications in material science and biology. At the moment we are trying to follow the motion of implanted beads in cells to measure the viscosity of the intracellular medium. Bacterial motion is next. On the technical side we hope to go to shorter wavelengths, hopefully into the $\mathrm{UV}$ or even soft $\mathrm{x}$-ray regime for the obvious reason of achieving higher resolution. And the list goes on and on.

\section{Acknowledgement}

This work was supported by grants from NSERC.

\section{References}

[1] Gabor, D. (1948) Nature (London) 161,777-778

[2] Gabor, D. 1949 Proc. R. Soc. London, Ser. A 197454.

[3] H.J. Kreuzer, M.H. Jericho, I.A. Meinertzhagen and Wenbo Xu. 2001 J. Phys.: Condens. Matter 13, 10729.

[3] Saxby, G. (1994) Practical Holography (Prentice Hall, 1994).

[5] Hariharan, P. (1996) Optical Holography (Cambridge University Press, Cambridge).

[6] Kreuzer, H.J., Nakamura, K., Wierzbicki, A., Fink, H.-W. and Schmid, H. 1992 Ultramicroscopy 45381.

[7] Kreuzer, H.J. and Pawlitzek, R.P. (1993-1998) LEEPS, Version 1.2, A software package for the simulation and reconstruction of Low Energy Electron Point Source images and other holograms.

[8] Xu, W., Jericho, M.H., Meinertzhagen, I.A., and Kreuzer, H.J. 2001 Proc. Natl. Acad. Sci. USA 98, 11301.

[9] Xu, W., Jericho, M.H., Meinertzhagen, I.A., and Kreuzer, H.J. 2002 Appl. Opt. 415367.

\section{Footnotes:}

${ }^{1}$ Members of the DIH group at Dalhousie University M.H. Jericho*, Wenbo $\mathrm{Xu}^{* * * *}$, and I.A. Meinertzhagen ${ }^{* *}$ ${ }^{*}$ Department of Physics and ${ }^{* *}$ Neuroscience Institute 